\title{
Brain Size Predicts the Success of Mammal Species Introduced into Novel Environments
}

\author{
Daniel Sol, ${ }^{1, *}$ Sven Bacher, ${ }^{2, \dagger}$ Simon M. Reader, ${ }^{3,4, \star}$ and Louis Lefebvre ${ }^{4, \S}$
}

1. Centre for Ecological Research and Applied Forestries (CREAF), Autonomous University of Barcelona, E-08193 Bellaterra, Catalonia, Spain;

2. Community Ecology, Zoological Institute, University of Bern, Baltzerstrasse 6, 3012 Bern, Switzerland;

3. Behavioural Biology and Helmholtz Institute, Utrecht

University, P.O. Box 80086, 3508 TB Utrecht, The Netherlands;

4. Department of Biology, 1205 Avenue Docteur Penfield, McGill

University, Montréal, Québec H3A 1B1, Canada

Online enhancement: appendix.

ABSTRACT: Large brains, relative to body size, can confer advantages to individuals in the form of behavioral flexibility. Such enhanced behavioral flexibility is predicted to carry fitness benefits to individuals facing novel or altered environmental conditions, a theory known as the brain size-environmental change hypothesis. Here, we provide the first empirical link between brain size and survival in novel environments in mammals, the largest-brained animals on Earth. Using a global database documenting the outcome of more than 400 introduction events, we show that mammal species with larger brains, relative to their body mass, tend to be more successful than species with smaller brains at establishing themselves when introduced to novel environments, when both taxonomic and regional autocorrelations are accounted for. This finding is robust to the effect of other factors known to influence establishment success, including introduction effort and habitat generalism. Our results replicate similar findings in birds, increasing the generality of evidence for the idea that enlarged brains can provide a survival advantage in novel environments.

Keywords: brain evolution, invasion biology, behavioral flexibility, propagule pressure, ecological generalism.

\footnotetext{
* Corresponding author; e-mail: d.sol@creaf.uab.es.

${ }^{\dagger}$ Present address: Ecology and Evolution Unit, University of Fribourg, Chemin du Musée 10, 1700 Fribourg, Switzerland; e-mail: sven.bacher@unifr.ch.

‡ E-mail: s.m.reader@uu.nl.

$\checkmark$ E-mail: louis.lefebvre@mcgill.ca.

Am. Nat. 2008. Vol. 172, pp. S63-S71. (C) 2008 by The University of Chicago. 0003-0147/2008/1720S1-42861\$15.00. All rights reserved.

DOI: $10.1086 / 588304$
}

Some mammals, such as primates and cetaceans, have evolved large brains despite the substantial metabolic and developmental costs involved (van Schaik and Deaner 2003; Isler and van Schaik 2006). Why? Past research on this question has centered on the brain's role in sensory, cognitive, and motor functions (Barton 2006). Increased brain size has been linked to sensory specializations (Joffe and Dunbar 1997; Barton 1998), cognitive skills (Reader and Laland 2002; Byrne and Corp 2004), and motor functions (Changizi 2003). Several of these associations have also been documented in birds (e.g., Lefebvre et al. 1997, 2004; Garamszegi et al. 2002), suggesting convergent evolution of the brain between these classes (Emery and Clayton 2004; Lefebvre et al. 2004). Although the underlying neurophysiological mechanisms are not well understood (Roth and Dicke 2005; Dunbar and Shultz 2007), the existence of such correlations suggests that enlarged brains enhance the capacities required for individuals to modify their behavior in potentially adaptive ways (Jerison 1973; Lefebvre et al. 1997; Allman 2000; Madden 2001; Reader 2003b; Barton 2004; Byrne and Corp 2004; Marino 2005).

Nonetheless, controversy remains regarding the advantages that enlarged brains provide for the survival and reproduction of animals in the wild (Marino 2005; Dunbar and Shultz 2007). A number of hypotheses have been put forward to explain such advantages, including the need to learn how to access hard-to-eat food (Parker and Gibson 1977), to track changes in the spatial or temporal distribution of food (Clutton-Brock and Harvey 1980), to deal with complex social interactions (Jolly 1966; Humphrey 1976; Sawaguchi and Kudo 1990; Byrne and Corp 2004; Shultz and Dunbar 2006; Dunbar and Shultz 2007; Lindefors et al. 2007), to use multiple foraging strategies (Ratcliffe et al. 2006), to discover novel resources (Lefebvre et al. 1997; Ratcliffe et al. 2006), and to deal with unpredictability in the environment (Richerson and Boyd 2000; Reader and MacDonald 2003). One principle unifying these hypotheses is the idea that enlarged brains afford advantages in dealing with environmental change when the response demands behavioral flexibility in the form of learning and innovation. Thus, some authors consider that large brains 
might function, and thus have evolved, to deal with novel socioecological challenges in general (Allman et al. 1993; Reader and Laland 2002; Marino 2005), a theory known as the brain size-environmental change hypothesis. However, evidence that enlarged brains provide a survival advantage when facing novel challenges is available only for birds. In birds, species with enlarged brains have been found to show higher survival in novel or altered environments (Sol and Lefebvre 2000; Shultz et al. 2005; Sol et al. 2005b) as well as in their native ranges (Sol et al. 2007), attributable to their enhanced behavioral flexibility.

Mammals are the taxonomic group that contains the largest brains of any animal on Earth, implying past selection on brain enlargement. Thus, mammals provide a valuable opportunity to test the generality of the brain size-environmental change hypothesis. Despite considerable interest in understanding the selection pressures acting on brain enlargement, there has been no attempt to validate the link between brain size and environmental change in mammals. Here, we address this important gap by asking whether large-brained mammals are more successful at surviving in novel environments. When exposed to a new environment, species will confront diverse challenges such as the discovery and adoption of novel resources, the avoidance of previously unknown predators, and the adjustment of behavior to a new social or physical environment (Duncan et al. 2003). The ability to rapidly cope with these new challenges through behavioral adjustments may be a matter of life or death (Mayr 1965; Morse 1980). Experimental introductions to novel regions are, in general, not logistically or ethically feasible in vertebrates, yet one can draw on the vast literature on past, human-mediated introductions for useful, relevant data. If the brain size-environmental change hypothesis is valid, we hypothesize that, other factors being equal, largebrained species should be more likely to become successfully established when introduced to regions outside their natural geographic range. We test this prediction with a global database of mammalian species introduced to new locations, employing modeling techniques that correct for problems associated with phylogenetic and spatial nonindependence among introductions. Because "other factors" are not always equal, we test the hypothesis in the light of alternative explanations shown to affect establishment success, including other clade attributes, introduction effort (e.g., number of individuals released), and features of the recipient community.

\section{Material and Methods}

\section{Introduction Data}

We compiled a global database of human-mediated introductions of mammals to new locations, drawn largely from
Long (2003) and references therein. The introduction of a species to a new location (an "introduction event") was defined as the release of individuals of a species to an island or a governmental state within a continental mainland that is outside the species' current native range. Following previous work (Blackburn and Duncan 2001a; Cassey et al. 2004; Sol et al. 2005b), we considered an introduction successful if it resulted in the establishment of a persistent population on the recipient island or state; it was considered unsuccessful otherwise. We excluded recent introductions (i.e., those that took place fewer than 20 years ago), for which it may be too early to determine whether a viable population has been established, and introductions for which the outcome (whether success or failure) was uncertain. Because the number of released individuals is a major determinant of establishment success (Lockwood et al. 2005), we restricted our analyses to introduction events where data on introduction effort were available, yielding a total of 513 introduction events on 100 species.

\section{Brain Measures}

We gathered data on brain mass for 3,076 adult specimens (or mean values of several specimens) of 1,635 species from a variety of sources (see appendix in the online edition of the American Naturalist). Where possible, original sources were consulted; however, where a later source combined new data with previously published data and it was not possible to separate the two, the later source was utilized. When information on brain mass was available from two or more sources, we estimated brain size by using the average brain mass of these different sources.

We evaluated the reliability of the measures of brain mass with a variance component analysis using species $(n=$ 407) for which information was available from two or more independent sources (mean number of sources $=3.7$ ). We compared the variation in brain mass (log transformed) within and between species while taking into account differences in body mass (also log transformed; see next paragraph for a justification for body size adjustments). Most variation $(92.9 \%)$ in relative brain mass was among rather than within species, suggesting that the measure is highly reliable. To further improve this reliability, we specified the number of different sources from which the brain mass was estimated in all the models that tested the relationship between brain size and establishment success. In addition, we also coded whether the brain was measured in a domestic or a wild animal, because domestic animals tend to have smaller brains compared to their wild ancestors (Kruska 2005). These two variables are not relevant for the purpose of this study and for simplicity are not presented in the final models.

In mammals, as in other vertebrates, larger species have 
larger brains (Jerison 1973; Martin 1981). The usual method to remove this allometric effect and hence obtain brain measures that are biologically meaningful (Deaner et al. 2000) is to calculate the residuals of a regression of $\log$ (brain mass) on log (body mass) (Deaner et al. 2000; Sol et al. 2005b). These residuals measure the extent to which the brain is either larger or smaller than expected for the species' body size. In mammals, however, the allometric line relating brain mass to body mass can differ across taxonomic groups, a phenomenon known as grade shifts. Grade shifts are problematic because they can affect the estimate of the allometric exponent, leading to biases in the calculation of the residuals (Pagel and Harvey 1988; Nunn and Barton 2000).

We dealt with the problem of grade shifts by estimating two different types of brain residuals. The first method estimates the residuals by fitting regression lines within the taxonomic order of the species ("taxonomically adjusted brain residuals" hereafter). We obtained the taxonomically adjusted residuals with a general linear model where the dependent variable was brain mass (log transformed) and the predictors were body mass (log transformed), the taxonomic order, and the interaction between the two. The residuals were calculated with information for all species $(n=663)$ from orders for which introduction data were available, regardless of whether the species was subject to introductions. These residuals are not directly comparable across orders, as they are calculated based on different allometric lines, but they can be compared using hierarchical modeling techniques (see "General Analyses"). Because taxonomically adjusted residuals measure the relative size of the brain of a species compared with those of other members of the same order, they also deal with the possibility that differences in brain size do not mean the same thing for different taxonomic orders, for example, as a consequence of brain structure differing between orders.

The second method we used to estimate the residuals is based on independent contrasts (Felsenstein 1985). Allometric regressions with phylogenetically independent contrasts are generally less affected by grade shifts because typically only a few contrasts will be affected, and these will have relatively little effect on the overall relation between contrasts (Nunn and Barton 2000). We used independent contrasts to obtain size-corrected species brains, using information for all species $(n=663)$ from orders for which introduction data were available. The alternative of basing residuals on only introduced species would carry the disadvantages of a decreased sample size and the risk of biasing the estimation of the allometric exponent. We computed size-corrected values for brain mass by following the general method of Blomberg et al. (2003) to deal with traits that vary closely with body size. First, we log transformed brain mass and body mass of each species and computed standardized phylogenetically independent contrasts for both traits, using the phylogeny proposed by Bininda-Emonds et al. (2007). Then, we computed a least squares linear regression through the origin for the contrasts and noted the slope, $b$ (allometric exponent). The relationship between contrasts of brain and body mass was strong $\left(R^{2}=0.93\right)$, and hence, the use of alternative line-fitting techniques would have minimal impact (Barton and Harvey 2000). We included a dummy variable that coded for the particular between-clade contrasts that were identified as "outliers" in the overall allometric relation, thus effectively "removing" grade shifts from the analysis and allowing a better estimate of the within-clade scaling relation. Finally, we computed sizecorrected values for brain mass (not contrasts) as $\log \left[\right.$ brain mass/(body mass $\left.\left.{ }^{b}\right)\right]$. The slope was estimated as $0.667 \pm 0.006(\mathrm{SE})$, very close to that estimated by Harvey and Krebs (1990). Because the residuals estimated in this way did not completely remove the effect of body size in our subsample of species with introduction data (correlation $=0.19$ ), we included log body mass in all the models testing the relationship between these residuals and establishment success.

The taxonomically adjusted brain residuals were significantly correlated with those estimated with independent contrasts (correlation coefficient $=0.80, P<.0001$ ) and yielded qualitatively similar results. We describe here the results obtained with the taxonomically adjusted residuals and present those obtained with the independent contrasts method in the appendix.

\section{Confounding Variables}

The success of species introductions is known to depend on the idiosyncrasies of the release event and the characteristics of the recipient community (Duncan et al. 2003). Such potentially confounding factors need to be controlled for in a proper test of the relationship between residual brain size and establishment success, because they could either inflate or obscure the predicted relationships. The most important event-level effects relate to introduction effort (Lockwood et al. 2005), which we recorded as the number of individuals released (Veltman et al. 1996; Cassey et al. 2004). In addition, we distinguished whether the introduction was deliberate or accidental.

Because some regions may be easier to invade than others, we also controlled for region-level effects by including biogeographical region as a variable in multivariate analyses. Based on the global pattern of introductions, we recognized six biogeographical regions: Australian, Indomalayan, Afrotropical, Neotropical, Palearctic, and Nearctic. Offshore volcanic islands (Hawaii, Society Islands, 
Caroline Islands, and Kerguelen Islands) were considered an additional category.

Besides event- and region-level effects, a number of species-level traits have been proposed or found to affect establishment success (Duncan et al. 2003; Sol 2007) and hence could confound the hypothesized link between brain residuals and establishment success. We examined the following traits: (1) habitat generalism (the number of habitat types a species has been reported to inhabit, out of a possible total of nine types [coastal habitats; inland surface waters; mires, bogs, and fens; grasslands; heathland, scrub, and tundra; woodland and forest; inland unvegetated or sparsely vegetated habitats; agricultural, horticultural, and domestic habitats; and constructed, industrial, and other artificial habitats]), (2) diet (herbivorous, carnivorous, or omnivorous), (3) annual fecundity (number of offspring per year, log transformed), (4) mating system (monogamous vs. alternative systems), (5) native geographic range size (estimated by summing the areas of countries or states/provinces in which the species is considered to be present), and (6) whether the introduction was on an island or on the mainland. All information was obtained from Grzimek (1988), except for geographic distribution (obtained from Wilson and Reeder 2005) and mating system (obtained from Komers and Brotherton 1997; Sæther and Gordon 1994; and the Animal Diversity Web, http://animaldiversity.ummz.umich.edu/site/index.html).

We finally considered the possibility that a species succeeds at establishing itself in a novel region not because it is preadapted to survive in novel environments in general but instead because it is preadapted to the specific region of introduction, the so-called climate-matching hypothesis (Williamson 1996). The degree of matching between the origin and introduction locations is difficult to evaluate at the global scale of this study, but one might expect the matching to be higher in introductions within the same biogeographical region of the species and when the species is introduced into a habitat that is similar to the one in its native range. Assessing whether an introduction took place within the same biogeographical region of the species was easy, but we often did not know the habitats to which the species were introduced, particularly for unsuccessful introductions. However, given that most introductions of mammals took place in man-made habitats, habitatmatching should be particularly important in species that occur in such habitats in their native ranges. We defined habitats that were strongly influenced by human activity as agricultural, gardens, parks, and urban habitats, which correspond to the last two categories defined above. We classified animals that have affiliations to humans as those that occur in these two habitat categories and included this new variable in the models to account for traitenvironment interactions.

\section{General Analyses}

Information on brain mass was available for 66 of the 100 introduced species that we considered (table A1 in the online edition of the American Naturalist), yielding a total of 443 introduction attempts for the analyses (table A2 in the online edition of the American Naturalist). In general, the analysis of historical introductions faces three major difficulties (Blackburn and Duncan 2001a; Sol et al., forthcoming). First, the sample of introduced species is likely to be a nonrandom subset of all mammal species, because species were generally transported and/or introduced for some purpose. Although the species used for introduction appear to be randomly selected with respect to their brain size (fig. 1), the absence of some taxonomic categories in our sample limits the generality of the results and needs to be accounted for in the analyses. Second, patterns of success are confounded because species were not randomly assigned to introduction locations and because some locations will have received disproportionately more invaders or more good or poor invaders (Blackburn and Duncan $2001 b$ ). For example, if large-brained species tend to have been introduced to regions that are easier to invade, then we could erroneously conclude that enlarged brains facilitate establishment in novel locations. Finally, individual introductions cannot be regarded as independent data points in a statistical analysis. Instead, introduction outcomes are likely to be correlated because most locations were subject to several introductions, the same species was frequently introduced to many locations, and species themselves are clustered due to phylogenetic relationships.

To deal with the above problems, we followed Blackburn and Duncan (2001a) and used GLIMMIX (SAS Institute, Cary, NC) to fit generalized linear mixed models. By including random effects in the model, GLIMMIX takes complex clustered structures of data into account during statistical analysis to provide estimates of standard error corrected for the resulting nonindependence. We modeled the likely nonindependence of introductions of closely related species by assuming a common positive correlation between introduction outcomes for species within nested taxonomic groups (species, families, and orders) but a zero correlation between introduction outcomes involving species in different groups (a variance components model). The use of taxonomy instead of a complete phylogeny is justified in our case because, as it is in birds (Blackburn and Duncan 2001a), variation in establishment success in mammals is mostly at the species level (see random factors in tables 1, A3 [in the online edition of the American Naturalist]), indicating low phylogenetic autocorrelation. Clustering of introduction events within biogeographical regions was similarly modeled. The remaining variables (residual brain and confounding variables) were included 


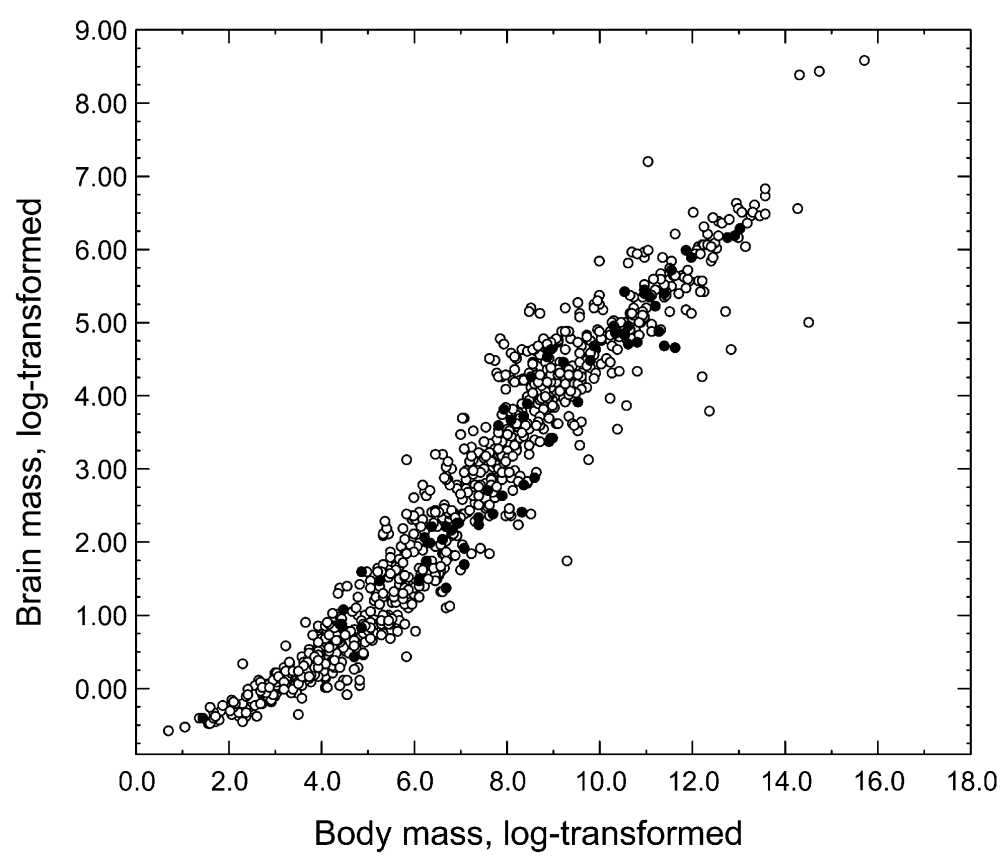

Figure 1: Brain mass of species used for introductions (solid circles) compared with brain mass of the rest of mammals (open circles). There are no differences between both samples of species in the intercept $(P=.75)$ or the slope $(P=.68)$ of the lines relating brain mass to body mass.

as fixed effects. Success was modeled by specifying a binomial error distribution and logit link function, with introduction outcome (establishment success or failure) as the response variable. Minimum adequate models were obtained by backward selection, removing nonsignificant fixed effects by using a conservative $P<.1$.

\section{Results}

In 343 out of 446 introduction attempts, mammals succeeded at establishing a persistent population in the region of introduction. Neither absolute brain mass nor body mass were correlated with establishment success $(P=.84$ and .73 , respectively) when both taxonomic and regional autocorrelations were accounted for. However, success was significantly higher for species with larger brains than for species with smaller brains once the allometric effect of body size was taken into account (GLMM: estimate $\pm \mathrm{SE}=$ $1.45 \pm 0.64, t=2.26, \mathrm{df}=40.7, P=.029)$. These results held when species with single introduction events and those belonging to the genus Cervus (the most frequently introduced genus) were removed $(P=.045)$. Analyses with residual brain size based on the regression line calculated using phylogenetic contrasts (see "Methods") provided qualitatively similar results (see table A3).

A number of event-, clade-, and region-level factors have been proposed or found to affect establishment
(Duncan et al. 2003; Sol 2007), and these effects need to be accounted for to properly test the brain size-environmental change hypothesis. Our analyses do not support a higher establishment success in species inhabiting humanmodified environments in their native range, or in those coming from the same biogeographic region (table 1). We also failed to find any effect of mating system or other life-history traits in species success in the novel environments (table 1). However, establishment success increased with the number of individuals released and the degree of habitat generalism of the species (tables 1, A3). When these confounding effects were controlled for in the analysis, residual brain size remained significantly associated with establishment success (tables 1, A3).

\section{Discussion}

Although there is considerable interest in the function and evolution of brain enlargement in vertebrates in general, evidence for the widely held idea that enlarged brains facilitate success in response to novel challenges arguably has been limited to birds. In birds, larger-brained, behaviorally innovative species are more likely to be successful in novel environments than are small-brained, less innovative species, a pattern described first in New Zealand (Sol and Lefebvre 2000) and then globally (Sol et al. 2005b). However, a critical part of developing and testing evolutionary theo- 
Table 1: Fixed and random effects in a generalized linear mixed model explaining variation in mammal establishment success while controlling for geographical region and taxonomic levels

\begin{tabular}{|c|c|c|c|c|c|c|c|c|}
\hline \multirow[b]{2}{*}{ Effect } & \multicolumn{4}{|c|}{ Full model $^{a}$} & \multicolumn{4}{|c|}{ Minimum adequate model ${ }^{\mathrm{b}}$} \\
\hline & Estimate & SE & $\begin{array}{c}\text { Type III, } \\
F\end{array}$ & $P$ & Estimate & SE & $\begin{array}{c}\text { Type III, } \\
\text { F }\end{array}$ & $P$ \\
\hline \multicolumn{9}{|l|}{ Fixed: } \\
\hline Introduction effort & .972 & .257 & 14.34 & .0002 & .938 & .237 & 15.62 & $<.0001$ \\
\hline Residual brain size & 2.250 & .792 & 8.07 & .0081 & 1.938 & .641 & 9.15 & .0049 \\
\hline Habitat generalism & .272 & .206 & 1.75 & .1952 & .294 & .161 & 3.34 & .0781 \\
\hline Body mass & .138 & .158 & .76 & .3889 & $\ldots$ & $\ldots$ & $\cdots$ & $\cdots$ \\
\hline Use of man-made habitats & .749 & .434 & 2.98 & .0911 & $\ldots$ & $\ldots$ & $\ldots$ & $\ldots$ \\
\hline Cause of introduction & -.092 to $.110^{c}$ & 1.696 & .07 & .9573 & $\ldots$ & $\ldots$ & $\ldots$ & $\ldots$ \\
\hline Fecundity & .080 & .174 & .21 & .6488 & $\ldots$ & $\ldots$ & $\ldots$ & $\ldots$ \\
\hline Introduction onto an island & .520 & .332 & 2.45 & .1181 & $\ldots$ & $\ldots$ & $\cdots$ & $\ldots$ \\
\hline Native range size & .128 & .433 & .09 & .7688 & $\cdots$ & $\cdots$ & $\cdots$ & $\ldots$ \\
\hline Introduction in the same biome & -.145 & .342 & .18 & .6719 & $\ldots$ & $\ldots$ & $\ldots$ & $\ldots$ \\
\hline Mating system & .313 & .782 & .16 & .6932 & $\ldots$ & $\ldots$ & $\ldots$ & $\ldots$ \\
\hline \multicolumn{9}{|l|}{ Random: } \\
\hline Orders & .000 & $\ldots$ & $\ldots$ & $\ldots$ & .000 & $\ldots$ & $\ldots$ & $\ldots$ \\
\hline Families within orders & .414 & .569 & $\ldots$ & $\ldots$ & .057 & .324 & $\cdots$ & $\cdots$ \\
\hline Species within families & .967 & .567 & $\ldots$ & $\ldots$ & .898 & .471 & $\ldots$ & $\ldots$ \\
\hline Biogeographical region & .000 & $\ldots$ & $\ldots$ & $\ldots$ & .058 & .130 & $\ldots$ & $\ldots$ \\
\hline
\end{tabular}

ries is to establish common, repeated patterns in different taxa (Marino 2005). Our finding that brain size is statistically associated with establishment success in mammals, the largest-brained vertebrates, extends previous findings and provides support for the generality of the brain size-environmental change hypothesis in vertebrates.

Our analyses focused on the relative size of the whole brain instead of the size of brain components, mainly for reasons of data availability. Our findings may result from whole-brain enlargement in particular species, from enlargement of large brain components, or from the correlated evolutionary enlargement of several brain areas. Thus, our analyses cannot contribute to the debate over whether whole-brain or part-brain measures best predict behavioral flexibility or cognitive capacity (Deaner et al. 2007). Contemporary studies of brain evolution have tended to focus on the size of particular areas of the brain, such as the neocortex, on the assumption that a focus on brain areas involved in the trait of interest is appropriate (Reader and Laland 2002; Deaner et al. 2007). However, such approaches do not invalidate analyses of whole-brain volume, for several reasons. First, many brain component volumes are tightly correlated with whole-brain volumes, particularly the large parts of the brain, such as the mammalian neocortex, that have received attention from students of primate cognitive evolution. Second, we propose that the link between brain size and introduction success that we document is the result of brain enlargement affording increases in the behavioral flexibility needed to deal with the variety of problems a species faces when exposed to a novel environment. Several measures of behavioral flexibility correlate with brain volume measures in primates (Reader and Laland 2002; Reader 2003a; Deaner et al. 2006) and bats (Ratcliffe et al. 2006). Behavioral flexibility involves a range of processes, including perception, motor, and cognitive processing (Reader and MacDonald 2003), and this processing is unlikely to be restricted to one brain area. Finally, brain areas perform many functions, and several brain areas may be involved in a given function. Moreover, "cognitive" capacities may, in reality, be difficult to disentangle from perceptual and motor processing (Barton 2006). Analysis of whether whole-brain or component volumes better predict introduction success is an empirical issue that can be addressed only when appropriate data become available.

According to the brain size-environmental change hypothesis, a large brain can have benefits in novel environments by facilitating adaptive behavioral responses to unusual or novel ecological challenges, through cognitive processes such as innovation, learning, and decision making. The importance of these cognitive processes in dealing with novel ecological challenges is supported in numerous studies 
(Dukas 1998, 2004; Sol, forthcoming). However, the mechanisms by which a large brain facilitates population persistence in novel environments through enhanced behavioral flexibility are less fully understood. Basically, there are two nonmutually exclusive routes to extinction in small invader populations (Sæther and Engen 2003). First, an introduced population may become extinct because of a negative mean population growth rate, for example, when individuals are incapable of finding appropriate resources in the new place or cannot deal with the pressure of novel enemies. A growing number of studies show that developing new behaviors and rapid learning are important for the discovery and adoption of novel resources and the avoidance of unfamiliar predators (Lefebvre et al. 1997; Brooker et al. 1998; Estes et al. 1998; Berger et al. 2001; Sol, forthcoming), suggesting that a large brain can facilitate success in novel environments by increasing the likelihood of finding an appropriate ecological niche.

Second, an invader population with a positive growth rate also may become extinct through the operation of demographic or environmental stochasticity affecting the population growth rate or fluctuations in the carrying capacity. The importance of demographic stochasticity in the invasion process is suggested in the finding that the size of the founder population is a main determinant of establishment success in vertebrates (Lockwood et al. 2005; this study). However, the influence of stochasticity should vary according to the life history of the species, the effect being less pronounced in species with low adult mortality (Pimm 1991; Legendre et al. 1999). If a large brain buffers individuals against ecological challenges posed by the environment, this should reduce mortality (Sol et al. 2007) and facilitate population persistence through a decrease in demographic stochasticity.

In the long term, the persistence of an introduced population will depend on the adaptation of the population to the novel environment (Mooney and Cleland 2001; Sakai et al. 2001). Local adaptation requires time, yet evolution can proceed extremely quickly in introduced populations facing strong directional selection (Baker and Moeed 1987; Reznick and Ghalambor 2001; Price 2008). This process might be accelerated in big-brained animals as a result of their enhanced ability to develop behavioral responses to novel selective pressures (Wyles et al. 1983; Robinson and Dukas 1999; Huey et al. 2003; Price et al. 2003; Price 2008; but see Huey et al. 2003 for an alternative model). Such behavioral responses can alter the intensities and/or directions of selection and reduce the probability that the population becomes extinct, facilitating adaptation to the novel environments through genetic assimilation (Price 2008). Support for the importance of brain size in evolution is scarce and limited to birds (Sol et al. 2005a), in which brain size is positively associated with intraspe- cific diversification independently of range expansion. In mammals, however, Lynch (1990) found no evidence that large-brained lineages evolved morphologically at a faster rate than small-brained ones.

The brain size-environmental change hypothesis is part of a more general theory, the cognitive buffer hypothesis, which suggests that larger and more complex brain function may have evolved to behaviorally buffer individuals against the vagaries of the external world (Allman et al. 1993; Deaner et al. 2003; Sol, forthcoming). The finding that brain size is associated with success in novel environments provides evidence for this buffer function of the brain, emphasizing that a large brain is particularly advantageous for the construction of adapted responses to novel ecological challenges. We have limited knowledge of the mechanisms by which the brain facilitates population persistence in the face of environmental changes, but understanding these mechanisms is likely to be an important avenue for future research.

\section{Acknowledgments}

We thank T. D. Price for the invitation to attend the Evolution 2007 invasive species symposium and to contribute to this special issue. We also thank K. Isler for providing the brain volume data utilized in Isler and van Schaik (2006), P. Abbegglen and A. Käser for compiling the introduction data, T. Garland and C. Nunn for valuable statistical advice, R. Deaner and R. Dunbar for sending us their papers, T. D. Price and three anonymous reviewers for useful comments on previous versions of this article, and T. Blackburn, P. Cassey, R. Duncan, S. Kark, J. Morand-Ferron, J. Pino, W. Solarz, M. Vall-Llosera, and M. Vilà for fruitful discussions over the past years. This work was supported by a Ramón y Cajal fellowship and a Proyecto de Investigación (CGL2007-66257) grant from the Ministerio de Educación y Ciencia (Spain), a travel grant from the American Society of Naturalists, and European Union Sixth Framework Programme integrated project ALARM (Assessing Large-Scale Environmental Risks for Biodiversity with Tested Methods) grant GOCE-CT-2003-506675.

\section{Literature Cited}

Allman, J. 2000. Evolving brains. Scientific American Library, New York.

Allman, J. M., T. McLaughlin, and A. Hakeem. 1993. Brain weight and life-span in primate species. Proceedings of the National Academy of Sciences of the USA 90:118-122.

Baker, A. J., and A. Moeed. 1987. Rapid genetic differentiation and founder effects in colonizing populations of common mynas (Acridotheres tristis). Evolution 41:525-538.

Barton, R. A. 1998. Visual specialization and brain evolution in primates. Proceedings of the Royal Society B: Biological Sciences 265: 1933-1937. 
2004. Binocularity and brain evolution in primates. Proceedings of the National Academy of Sciences of the USA 101: 10113-10115.

- 2006. Primate brain evolution: integrating comparative, neurophysiological, and ethological data. Evolutionary Anthropology 15:224-236.

Barton, R. A., and P. H. Harvey. 2000. Mosaic evolution of brain structure in mammals. Nature 405:1055-1058.

Berger, J., J. E. Swenson, and I.-L. Persson. 2001. Recolonizing carnivores and naive prey: conservation lessons from Pleistocene extinctions. Science 291:1036-1039.

Bininda-Emonds, O. R. P., M. Cardillo, K. E. Jones, R. D. E. MacPhee, R. M. D. Beck, R. Grenyer, S. A. Price, et al. 2007. The delayed rise of present-day mammals. Nature 446:507-512.

Blackburn, T. M., and R. P. Duncan. 2001a. Determinants of establishment success in introduced birds. Nature 414:195-197.

- 2001b. Establishment patterns of exotic birds are constrained by non-random patterns in introduction. Journal of Biogeography 28:927-939.

Blomberg, S. P., T. J. Garland, and A. R. Ives. 2003. Testing for phylogenetic signal in comparative data: behavioral traits are more labile. Evolution 57:717-745.

Brooker, M. L., N. B. Davies, and D. G. Noble. 1998. Rapid decline of host defences in response to reduced cuckoo parasitism: behavioural flexibility of reed warblers in a changing world. Proceedings of the Royal Society B: Biological Sciences 265:1277-1282.

Byrne, R. W., and N. Corp. 2004. Neocortex size predicts deception rate in primates. Proceedings of the Royal Society B: Biological Sciences 271:1693-1699.

Cassey, P., T. M. Blackburn, D. Sol, R. P. Duncan, and J. L. Lockwood. 2004. Global patterns of introduction effort and establishment success in birds. Proceedings of the Royal Society B: Biological Sciences 271:S405-S408.

Changizi, M. 2003. Relationship between number of muscles, behavioral repertoire size, and encephalization in mammals. Journal of Theoretical Biology 220:157-168.

Clutton-Brock, T. H., and P. H. Harvey. 1980. Primates, brains and ecology. Journal of Zoology (London) 190:309-323.

Deaner, R. O., C. L. Nunn, and C. P. van Schaik. 2000. Comparative tests of primate cognition: different scaling methods produce different results. Brain, Behavior and Evolution 55:44-52.

Deaner, R. O., R. A. Barton, and C. P. van Schaik. 2003. Primate brains and life histories: renewing the connection. Pages 233-265 in P. M. Kappeler and M. E. Pereira, eds. Primates life histories and socioecology. University of Chicago Press, Chicago.

Deaner, R. O., C. P. Van Schaik, and V. Johnson. 2006. Do some taxa have better domain-general cognition than others? a meta-analysis of nonhuman primate studies. Evolutionary Psychology 4:149-196.

Deaner, R. O., K. Isler, J. Burkart, and C. P. van Schaik. 2007. Overall brain size, and not encephalization quotient, best predicts cognitive ability across non-human primates. Brain, Behavior and Evolution 70:115-124.

Dukas, R. 1998. Evolutionary ecology of learning. Pages 129-174 in R. Dukas, ed. Cognitive ecology: the evolutionary ecology of information processing and decision making. University of Chicago Press, Chicago.

- 2004. Evolutionary biology of animal cognition. Annual Review of Ecology and Systematics 35:347-374.

Dunbar, R. I. M., and S. Shultz. 2007. Understanding primate brain evolution. Philosophical Transactions of the Royal Society B: Biological Sciences 352:649-658.

Duncan, R. P., T. M. Blackburn, and D. Sol. 2003. The ecology of avian introductions. Annual Review of Ecology, Evolution and Systematics 34:71-98.

Emery, N. J., and N. S. Clayton. 2004. The mentality of crows: convergent evolution of intelligence in corvids and apes. Science 306: 1903-1907.

Estes, J. A., M. T. Tinker, T. M. Williams, and D. F. Doak. 1998. Killer whale predation on sea otters linking oceanic and nearshore ecosystems. Science 282:473-476.

Felsenstein, J. 1985. Phylogenies and the comparative method. American Naturalist 125:1-15.

Garamszegi, L. Z., A. P. Møller, and J. Erritzoe. 2002. Coevolving avian eye size and brain size in relation to prey capture and nocturnality. Proceedings of the Royal Society B: Biological Sciences 269:961-967.

Grzimek, B. 1988. Enzyklopädie Säugetiere. Vols. 1-11. Kindler, Munich.

Harvey, P. H., and J. R. Krebs. 1990. Comparing brains. Science 249: 140-145.

Huey, R. B., P. E. Hertz, and B. Sinervo. 2003. Behavioral drive versus behavioral inertia in evolution: a null model approach. American Naturalist 161:357-366.

Humphrey, N. K. 1976. The social function of intellect. Pages $303-$ 317 in P. P. G. Bateson and R. A. Hinde, eds. Growing points in ethology. Cambridge University Press, Cambridge.

Isler, K., and C. P. van Schaik. 2006. Metabolic costs of brain size evolution. Biology Letters 2:557-560.

Jerison, H. J. 1973. Evolution of the brain and intelligence. Academic Press, New York.

Joffe, T. H., and R. I. M. Dunbar. 1997. Visual and socio-cognitive information processing in primate brain evolution. Proceedings of the Royal Society B: Biological Sciences 264:1303-1307.

Jolly, A. 1966. Lemur social behavior and primate intelligence. Science 153:501-506.

Komers, P. E., and P. N. M. Brotherton. 1997. Female space use is the best predictor of monogamy in mammals. Proceedings of the Royal Society B: Biological Sciences 264:1261-1270.

Kruska, D. C. T. 2005. On the evolutionary significance of encephalization in some eutherian mammals: effects of adaptive radiation, domestication, and feralization. Brain, Behavior and Evolution 65: 73-108.

Lefebvre, L., P. Whittle, E. Lascaris, and A. Finkelstein. 1997. Feeding innovations and forebrain size in birds. Animal Behaviour 53:549560.

Lefebvre, L., S. M. Reader, and D. Sol. 2004. Brains, innovations and evolution in birds and primates. Brain, Behavior and Evolution 63:233-246.

Legendre, S., J. Clobert, A. P. Møller, and G. Sorci. 1999. Demographic stochasticity and social mating system in the process of extinction of small populations: the case of passerines introduced to New Zealand. American Naturalist 153:449-463.

Lindefors, P., C. L. Nunn, and R. A. Barton. 2007. Primate brain architecture and selection in relation to sex. BMC Biology 5:20.

Lockwood, J. L., P. Cassey, and T. M. Blackburn. 2005. The role of propagule pressure in explaining species invasions. Trends in Ecology \& Evolution 20:223-228.

Long, J. L. 2003. Introduced mammals of the world: their history, distribution, and influence. CSIRO, Collingwood. 
Lynch, M. 1990. The rate of morphological evolution in mammals from the standpoint of the neutral expectation. American Naturalist 136:727-741.

Madden, J. 2001. Sex, bowers and brains. Proceedings of the Royal Society B: Biological Sciences 268:833-838.

Marino, L. 2005. Big brains do matter in new environments. Proceedings of the National Academy of Sciences of the USA 102: 5306-5307.

Martin, R. D. 1981. Relative brain size and basal metabolic rate in terrestrial vertebrates. Nature 293:57-60.

Mayr, E. 1965. The nature of colonising birds. Pages 29-43 in H. G. Baker, and G. L. Stebbins, eds. The genetics of colonizing species. Academic Press, New York.

Mooney, H. A., and E. E. Cleland. 2001. The evolutionary impact of invasive species. Proceedings of the National Academy of Sciences of the USA 98:5446-5451.

Morse, D. H. 1980. Behavioral mechanisms in ecology. Harvard University Press, Cambridge, MA.

Nunn, C. L., and R. A. Barton. 2000. Allometric slopes and independent contrasts: a comparative test of Kleiber's law in primate ranging patterns. American Naturalist 156:519-533.

Pagel, M. D., and P. H. Harvey. 1988. The taxon-level problem in the evolution of mammalian brain size: facts and artifacts. American Naturalist 132:344-359.

Parker, S. T., and K. R. Gibson. 1977. Object manipulation, tool use and sensorimotor intelligence as feeding adaptations in cebus monkeys and great apes. Journal of Human Evolution 6:623-641.

Pimm, S. L. 1991. The balance of nature? University of Chicago Press, Chicago.

Price, T. D. 2008. Speciation in birds. Roberts, Boulder, CO.

Price, T. D., A. Qvarnström, and D. E. Irwin. 2003. The role of phenotypic plasticity in driving genetic evolution. Proceedings of the Royal Society B: Biological Sciences 270:1433-1440.

Ratcliffe, R. M., M. B. Fenton, and S. J. Shettleworth. 2006. Behavioral flexibility positively correlated with relative brain volume in predatory bats. Brain, Behavior and Evolution 67:165-176

Reader, S. M. 2003a. Innovation and social learning: individual variation and brain evolution. Animal Biology 53:147-158.

2003b. Relative brain size and the distribution of innovation and social learning across the nonhuman primates. Pages 56-93 in D. Fragaszy and S. Perry, eds. The biology of traditions: models and evidence. Cambridge University Press, Cambridge.

Reader, S. M., and K. N. Laland. 2002. Social intelligence, innovation, and enhanced brain size in primates. Proceedings of the National Academy of Sciences of the USA 99:4436-4441.

Reader, S. M., and K. MacDonald. 2003. Environmental variability and primate behavioural flexibility. Pages $83-116$ in S. M. Reader and K. N. Laland, eds. Animal innovation. Oxford University Press, Oxford.

Reznick, D. N., and C. K. Ghalambor. 2001. The population ecology of contemporary adaptations: what empirical studies reveal about the conditions that promote adaptive evolution. Genetica 112113:183-198.

Richerson, P. J., and R. Boyd. 2000. Climate, culture and the evolution of cognition. Pages 329-346 in C. Heyes, and L. Huber, eds. The evolution of cognition. MIT Press, Cambridge, MA.

Robinson, B. W., and R. Dukas. 1999. The influence of phenotypic modifications on evolution: the Baldwin effect and modern perspectives. Oikos 85:582-589.
Roth, G., and U. Dicke. 2005. Evolution of the brain and intelligence. Trends in Cognitive Sciences 9:250-257.

Sæther, B.-E., and S. Engen. 2003. Routes to extinction. Pages 218236 in T. M. Blackburn and K. J. Gaston, eds. Macroecology: concepts and consequences. Blackwell, Oxford.

Sæther, B.-E., and I. J. Gordon. 1994. The adaptive significance of reproductive strategies in ungulates. Proceedings of the Royal Society B: Biological Sciences 256:263-268.

Sakai, A. K., F. W. Allendorf, J. S. Holt, D. M. Lodge, J. Molofsky, K. A. With, S. Baughman, et al. 2001. The population biology of invasive species. Annual Review of Ecology and Systematics 32: 305-332.

Sawaguchi, T., and H. Kudo. 1990. Neocortical development and social structure in primates. Primates 31:283-290.

Shultz, S., and R. I. M. Dunbar. 2006. Both social and ecological factors predict ungulate brain size. Proceedings of the Royal Society B: Biological Sciences 273:207-215.

Shultz, S., R. Bradbury, K. Evans, R. D. Gregory, and T. M. Blackburn. 2005. Brain size and resource specialisation predict long-term population trends in British birds. Proceedings of the Royal Society B: Biological Sciences 272:2305-2311.

Sol, D. 2007. Do successful invaders exist? Pre-adaptations to novel environments in terrestrial vertebrates. Pages 127-141 in W. Nentwig, ed. Biological invasions. Ecological Studies. Springer, Heidelberg.

. Forthcoming. The cognitive-buffer hypothesis for the evoution of large brains. In R. Dukas, and R. M. Ratcliffe, eds. Cognitive ecology. University of Chicago Press, Chicago.

Sol, D., and L. Lefebvre. 2000. Behavioural flexibility predicts invasion success in birds introduced to New Zealand. Oikos 90:599-605.

Sol, D., D. G. Stirling, and L. Lefebvre. 2005a. Behavioral drive or behavioral inhibition in evolution: subspecific diversification in Holarctic passerines. Evolution 59:2669-2677.

Sol, D., R. P. Duncan, T. M. Blackburn, P. Cassey, and L. Lefebvre. 2005b. Big brains, enhanced cognition, and response of birds to novel environments. Proceedings of the National Academy of Sciences of the USA 102:5460-5465.

Sol, D., T. Szekely, A. Liker, and L. Lefebvre. 2007. Big-brained birds survive better in nature. Proceedings of the Royal Society B: Biological Sciences 274:763-769.

Sol, D., M. Vilà, and I. Kühn. 2007. The comparative analysis of historical alien introductions. Biological Invasions, DOI: 10.1007/ s10530-007-9189-7.

van Schaik, C. P., and R. O. Deaner. 2003. Life history and cognitive evolution in primates. Pages 5-25 in F. B. M. de Waal and P. L. Tyack, eds. Animal social complexity. Harvard University Press, Cambridge, MA.

Veltman, C. J., S. Nee, and M. J. Crawley. 1996. Correlates of introduction success in exotic New Zealand birds. American Naturalist 147:542-557.

Williamson, M. H. 1996. Biological invasions. Chapman \& Hall, London.

Wilson, D. E., and D. M. Reeder. 2005. Mammal species of the world. Johns Hopkins University Press, Baltimore.

Wyles, J. S., J. G. Kunkel, and A. C. Wilson. 1983. Birds, behavior and anatomical evolution. Proceedings of the National Academy of Sciences of the USA 80:4394-4397.

Symposium Editor: Trevor D. Price 